\title{
Topography-Dependent Motion Compensation for Repeat-Pass Interferometric SAR Systems
}

\author{
Pau Prats, Student Member, IEEE, Andreas Reigber, Member, IEEE, and Jordi J. Mallorqui, Member, IEEE
}

\begin{abstract}
This letter presents a new motion compensation algorithm to process airborne interferometric repeat-pass synthetic aperture radar (SAR) data. It accommodates topography variations during SAR data processing, using an external digital elevation model. The proposed approach avoids phase artifacts, azimuth coregistration errors, and impulse response degradation, which usually appear due to the assumption of a constant reference height during motion compensation. It accurately modifies phase history of all targets before azimuth compression, resulting in an enhanced image quality. Airborne L-band repeat-pass interferometric data of the German Aerospace Center experimental airborne SAR (E-SAR) is used to validate the algorithm.
\end{abstract}

Index Terms-Calibration, image registration, interferometry, motion compensation, repeat-pass interferometry, synthetic aperture radar (SAR).

\section{INTRODUCTION}

H IGH-PRECISION airborne synthetic aperture radar (SAR) data processing is limited because of two main reasons: the lack of accuracy in the measurement of the antenna positions during data acquisition, and the assumption of a constant reference height during motion compensation (MoCo). Both are causing similar effects in the final compressed image, as both introduce uncompensated motion errors in the phase history of a target. In [1], an efficient algorithm to estimate and correct the former problem is proposed. This letter discusses a solution for the second one.

MoCo is usually carried out by assuming a reference level to compute the range displacements and phase corrections to apply to each received echo. This means that phase histories of targets at heights different from the reference level can not be matched accurately, which might yield several effects in the final compressed image [2]. Considering residual phase errors up to quadratic order, constant values will induce phase artifacts, linear components an azimuth shift of the impulse response, and

Manuscript received November 12, 2004; revised January 17, 2005. This work was supported in part by the Spanish MCYT and FEDER funds under project TIC2003-04451-C02-01 and in part by the Spanish-German Integrated Action, financed by MCYT (HA2003-0113) and DAAD (D/03/40325), respectively.

P. Prats is with the Department of Signal Theory and Communications, Universitat Politècnica de Catalunya (UPC), Barcelona E-08034, Spain, and also with the Department of Telecommunications and Engineering Systems, Universitat Autònoma de Barcelona (UAB), Bellaterra E-08193, Spain (e-mail: pprats@tsc.upc.edu).

A. Reigber is with the Department of Computer Vision and Remote Sensing, Technical University Berlin, Berlin 10623, Germany (e-mail: anderl@fpk.tuberlin.de).

J. J. Mallorqui is with the Department of Signal Theory and Communications, Universitat Politècnica de Catalunya (UPC), Barcelona E-08034, Spain (e-mail: mallorqui@tsc.upc.edu).

Digital Object Identifier 10.1109/LGRS.2005.846005 quadratic ones resolution loss plus phase artifacts. Higher order terms will further degrade the azimuth impulse response and phase quality. Thus, the computed interferogram will possess significant phase and azimuth coregistration errors. Several publications propose approaches to correct phase aberrations [2], [3], but all of them suggest the correction to be applied only for the beam-center position, not taking into account the whole synthetic aperture. In case of topography, the computed phase corrections might be incorrect and the aforementioned effects are not corrected precisely.

In this letter, an efficient way to use the information given by an external digital elevation model (DEM) to take into account the motion of the aircraft along the whole synthetic aperture is presented. It allows to obtain accurate interferometric phase estimates, and at the same time avoids azimuth coregistration errors and impulse response degradation. The algorithm uses a similar technique as in [4], so that using subapertures in time domain allows accurate topography accommodation. The solution can be easily implemented inside the structure of computationally efficient SAR processors like range-Doppler and chirp scaling (CSA) algorithms, meanwhile only modified approaches of $\omega-\mathrm{k}$ [5], [6] allow for this correction.

In Section II, an overview is presented, showing the limitations of repeat-pass systems in delivering high-quality phase products of areas with strong topography when using conventional MoCo techniques. Section III expounds the new MoCo approach. Finally, Section IV presents some results with data acquired by the German Aerospace Center (DLR) experimental airborne SAR (E-SAR).

\section{Limitations In CONVENTIONAL REPEAT-PASS INTERFEROMETRIC MOCO}

Assuming that range compression and range cell migration correction (RCMC) have been applied, the signal in time domain for a given target has the following expression (a squint of 0 has been assumed for the sake of simplicity):

$$
\begin{aligned}
& s\left(t, \tau ; r_{0}\right)=\zeta \cdot \exp \left[-j \frac{4 \pi}{\lambda} \sqrt{r_{0}^{2}+v^{2} \cdot\left(t-t_{0}\right)^{2}}\right] \\
& \cdot \exp \left[-j \frac{4 \pi}{\lambda} \varepsilon\left(t ; r_{0}\right)\right] \cdot s_{a}\left(t-t_{0}\right) \cdot s_{r c}\left(\tau-\frac{2 r_{0}}{c}\right)
\end{aligned}
$$

where $\zeta$ is a complex constant, $t$ is the azimuth time, $\tau$ is the range time, $r_{0}$ is the closest approach distance, $v$ is the forward velocity of the platform, $t_{0}$ is the zero-Doppler time position, $c$ is the speed of light, $\varepsilon\left(t ; r_{0}\right)$ is the uncorrected trajectory for that target, and $s_{a}(\cdot)$ and $s_{r c}(\cdot)$ are the azimuth and range compressed envelopes, respectively. With range-Doppler and chirp 
TABLE I

MAIN SySTEM AND PROCESSING PARAMETERS

\begin{tabular}{lc}
\hline System $P R F$ & $400 \mathrm{~Hz}$ \\
Sampling frequency & $100 \mathrm{MHz}$ \\
Processed azimuth bandwidth & $100 \mathrm{~Hz}$ \\
Processed range bandwidth & $75 \mathrm{MHz}$ \\
Wavelength & $0.2305 \mathrm{~m}$ \\
Near range & $3105 \mathrm{~m}$ \\
Far range & $5581 \mathrm{~m}$ \\
Mean flight altitude & $2600 \mathrm{~m}$ \\
Mean forward velocity & $95 \mathrm{~m} / \mathrm{s}$ \\
Effective baseline & $3.5 \mathrm{~m}$ \\
\hline
\end{tabular}

scaling algorithms, a two-step MoCo is commonly applied [7], where first-order MoCo corrects both envelope and phase for a reference range and height, while second-order MoCo corrects for each range after RCMC and range compression. Therefore, second-order MoCo is carried out multiplying (1) by a complex function containing the residual range-dependent correction $\varepsilon_{h 0}\left(t ; r_{0}\right)$, where subscript $h 0$ means the correction is made assuming a constant reference height. If the introduced term is equal to the error $\varepsilon\left(t ; r_{0}\right)$, MoCo is applied correctly, i.e., the height of the target is equal to the reference one used during second-order MoCo. However, this is normally not the case if strong topography variations are present in the scene. Therefore, a phase error remains along the phase history of the target, which, after azimuth compression, yields to phase errors and both degradation and displacement of the impulse response along azimuth direction. Consequently, although conventional MoCo has been applied, the error depends on the topography, making azimuth compression still space-variant. The main problem to overcome is the fact that for a given pulse, it is not possible to correct for more than one height. The subaperture approach presented in Section III expounds a solution to this problem.

The phase offset value due to second-order MoCo mismatch can be evaluated analytically for the maximum of the impulse response. Assuming time domain azimuth compression, i.e., a cross-correlation, the expression for instant $t_{0}$ is

$$
\begin{array}{r}
s_{c}\left(t_{0}, \tau ; r_{0}\right)=\zeta \cdot \exp \left[-j \frac{4 \pi}{\lambda} r_{0}\right] \cdot s_{r c}\left(\tau-\frac{2 r_{0}}{c}\right) \cdot \int_{L_{\mathrm{sa}}} s_{a}\left(t^{\prime}-t_{0}\right) \\
\cdot \exp \left[-j \frac{4 \pi}{\lambda} \varepsilon\left(t^{\prime} ; r_{0}\right)\right] \cdot \exp \left[j \frac{4 \pi}{\lambda} \varepsilon_{h 0}\left(t^{\prime} ; r_{0}\right)\right] \cdot \mathrm{d} t^{\prime} \quad(2)
\end{array}
$$

where $L_{\mathrm{sa}}$ is the length of the synthetic aperture in seconds. The integral in (2) should have a zero phase value. In any other case, an offset is introduced in the interferometric phase

$$
\phi=\frac{4 \pi}{\lambda} \Delta r+\phi_{\varepsilon}^{m}-\phi_{\varepsilon}^{s}
$$

where the first term contains the topographic information, and $\phi_{\varepsilon}^{m}, \phi_{\varepsilon}^{s}$ are the result of the integral for master and slave images, respectively. The integral in (2) can be computed numerically for some given $\varepsilon\left(t ; r_{0}\right)$ and $\varepsilon_{h 0}\left(t ; r_{0}\right)$. With the values of Table I and using the real trajectories, a target at an incidence angle of $45^{\circ}$ with a height of $100 \mathrm{~m}$ over the reference height will have a phase error of $\sim 400^{\circ}$.
Ideally, a solution would be to subtract the contribution of $\varepsilon_{h 0}^{x}$ from $\phi_{\varepsilon}^{x}$, where superscript $x$ refers to master and slave channels, respectively. However, this is not possible, because $\phi_{\varepsilon}^{x}$ is the result of a sum of weighted complex exponentials, and its computation is not possible without knowing $\varepsilon^{x}\left(t ; r_{0}\right)$. Solutions proposed in literature [2], [3] just take the value of $\varepsilon_{h 0}\left(t ; r_{0}\right)$ at beam-center position to perform a first-order correction. This solution leads to wrong results when $\varepsilon\left(t ; r_{0}\right)$ (and therefore $\left.\varepsilon_{h 0}\left(t ; r_{0}\right)\right)$ is not constant along the synthetic aperture. Therefore, an important conclusion is that, when having strong topography variations within the scene, DEMs derived from repeat-pass airborne InSAR systems may contain significant height errors when variations in the topography are not considered during MoCo. Additionally to phase errors, also coregistration errors and degradation of the impulse response are occurring in this case. Note that in single-pass interferometry, MoCo corrections are mostly the same for both master and slave channels, i.e., motion errors are correlated, so the introduced offset in the interferogram and coregistration errors along azimuth are practically null [2].

With the use of an external DEM, one could think of computing the integral in (2) for each pixel of the image and for both master and slave tracks, and correct the interferogram afterward. However, this would not prevent the other effects mentioned above. The next section expounds a solution that avoids all harmful effects by modifying the phase history of targets accurately before azimuth compression.

\section{TOPOGRAPHY-DEPENDENT MOTION COMPENSATION USING SUBAPERTURES}

\section{A. Basic Principle}

A first solution could consist in computing conventional second-order MoCo using the height information of an external DEM. This solution has the drawback that the correction is only applied using one height, which could be the mean height of the antenna footprint for each range for that pulse, and thus not being able to accommodate for other heights. To put some numbers, a system with the parameters of Table I would have a synthetic aperture of $\sim 530 \mathrm{~m}$ in midrange. If the observed scene had strong topography variations, the correction would result insufficient.

The algorithm proposed in this letter allows for an angle-accommodation following a similar principle than the one presented in [4], but taking into account topography. Fig. 1 shows the block diagram of the proposed algorithm. The idea is that taking small blocks along azimuth dimension in time domain, and applying short-time Fourier transforms (STFT) along that same dimension, allows for a time-frequency (or time-angle) dependent correction. With this principle, the authors of [4] were able to apply accurate MoCo to low-frequency wide-beam data. A step further is to apply a topography-dependent correction using the same principle. For a given block size and after azimuth STFT, the relation between azimuth frequencies and azimuth angles along the beam is given by the Doppler formula

$$
\beta(n)=\sin ^{-1}\left(\frac{\lambda \cdot f_{a}(n)}{2 \cdot v}\right)
$$




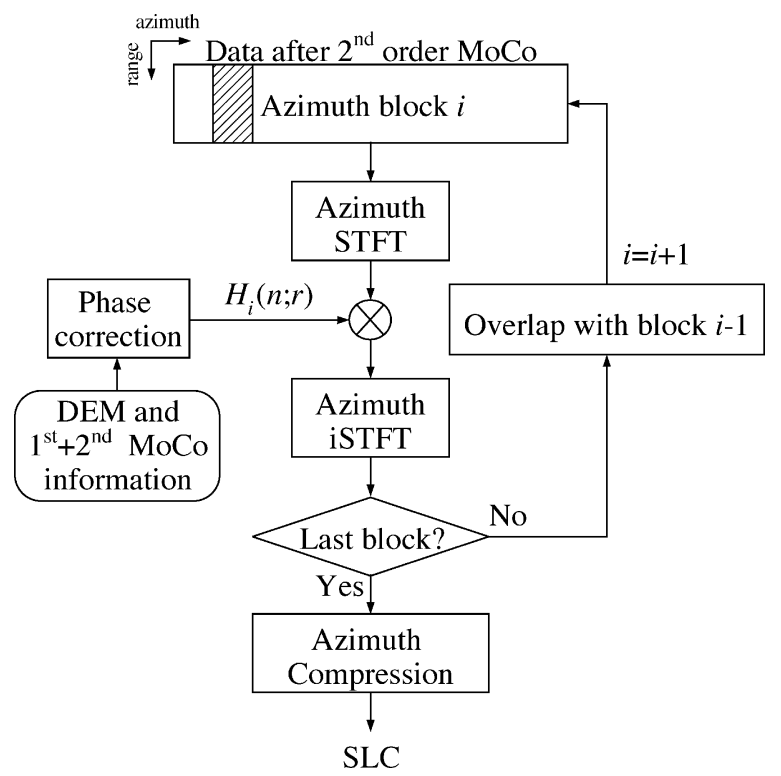

Fig. 1. Block diagram of the proposed topography-dependent MoCo algorithm.

where $n$ refers to vector position, and $f_{a}(n)$ is the azimuth frequency axis. Equation (4) means that a certain azimuth frequency corresponds to targets seen from the platform at a certain azimuth angle $\beta(n)$. The mapping between these angles and azimuth positions is

$$
x(n)=x_{0_{i}}+r_{0} \cdot \tan [\beta(n)]
$$

where $x_{0_{i}}$ is the azimuth center position of subaperture $i$. Therefore, knowing the azimuth position and with the use of a DEM back-geocoded to slant-range geometry, it is possible to know all three coordinates in space of the target and, consequently, to compute the correct MoCo phase. The proposed subaperture algorithm should be applied after conventional second-order MoCo, because at that stage, range compression has already been applied (note that in [4] the correction is applied before range compression). Therefore, it is possible to make the following residual range-dependent phase correction

$$
H_{i}\left(n ; r_{0}\right)=\exp \left[-j \frac{4 \pi}{\lambda}\left(-r_{\text {real }}(n)+\frac{r_{0}}{\cos [\beta(n)]}-r_{\mathrm{mc}_{i}}\right)\right]
$$

where $r_{\text {real }}(n)$ is the true distance to the target computed using the DEM and taking into account $\beta(n) . r_{\mathrm{mc}_{i}}$ is the total MoCo correction already applied to the center of subaperture $i$, i.e., first- plus second-order MoCo corrections. Notice that (6) is applied in the range-Doppler domain as depicted in Fig. 1. Finally, the subaperture is inverse Fourier transformed along azimuth and stored, before continuing with the next azimuth subaperture. It is interesting to note that (6) is also taking into account angle accommodation along the beam as in [4].

Using again the parameters of Table I and a block size of 64 samples, the resolution during MoCo along azimuth dimension is of about $8 \mathrm{~m}$, which allows for an accurate accommodation of topography variations.

\section{B. Practical Considerations}

Due to the subaperture philosophy of the algorithm, phase jumps along the phase history of targets might introduce severe sidelobes in the impulse response. A first step to reduce phase jumps is to apply second-order MoCo with a reference height given by the mean height of the antenna footprint for each range and for every pulse, instead of using one reference height for the whole image. More important, sidelobes should be further reduced by applying overlap between subapertures with a linear weighting where they overlap.

The performance of the proposed algorithm depends on the accuracy of the external DEM. Therefore, the phase error after the correction will have the same dependence as the one shown in (2). For the example given, an error in the DEM of $15 \mathrm{~m}$ will induce a phase error of about $10^{\circ}$.

The length of the subaperture is another key factor. A longer subaperture allows more resolution in frequency domain, thus accommodating topography with more accuracy. However, in case motion errors might vary noticeably along the subaperture, (6) becomes less accurate. Also, a longer subaperture implies that less corrections along the synthetic aperture are made. Values for the blocksize in azimuth between 32 or 64 have proven to yield good results for the azimuth processing bandwidth shown in Table I.

Results presented in next section use a 90-m resolution Shuttle Radar Topography Mission (SRTM) (http://www.jpl.nasa.gov/srtm/) back-geocoded DEM to apply the proposed topography-dependent MoCo algorithm. Because they are available to public, they become an excellent choice if no other DEMs with higher resolution are available. Results can be refined with a second iteration using the computed DEM. SRTM data were interpolated with efficient cubic convolution algorithms in order to obtain a smoothed back-geocoded DEM with the desired pixel spacing. Linear interpolation resulted in phase artifacts, caused by the subaperture philosophy of the proposed approach.

\section{RESULTS}

To validate the proposed method, with any loss of generality, airborne repeat-pass E-SAR data were used. The observed scene is located in south Germany near Bad Feilnbach. Data were processed with both CSA [8] and a time-domain backprojection algorithm. The latter is used in order to assess the accuracy of the technique as it focuses the image without approximations, i.e., its result is the ideal solution. The drawback in this case is, of course, the computation burden. The performance of the proposed approach can be evaluated by comparing both outputs. An SRTM DEM of the area was used with both processors for MoCo and range coregistration, the latter for interferometric purposes. Fig. 2 shows the SAR amplitude and the SRTM back-geocoded DEM of the observed scene, with dimensions $5.4 \times 2.2 \mathrm{~km}$. Table I shows the main system and processing parameters. For the CSA case, both master and slave data were processed in the following three ways:

1) with conventional two-step MoCo, i.e., using a constant reference height in both steps; 


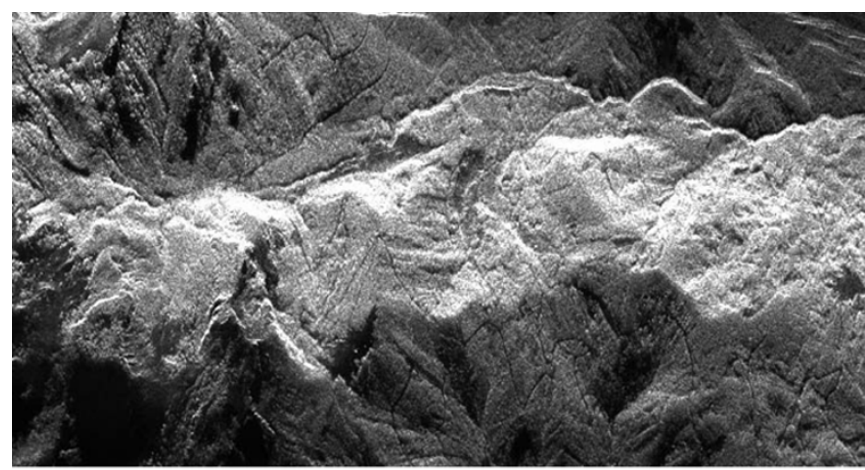

(a)

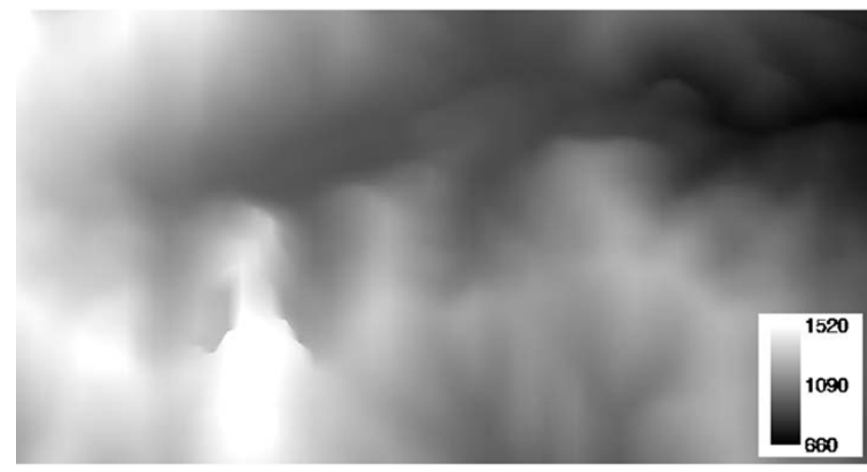

(b)

Fig. 2. (a) Amplitude and (b) back-geocoded SRTM DEM in meters over the WGS-84 ellipsoid of the observed scene. Azimuth is horizontal, and range is vertical, with near range on the top of the image.

2) same as 1), but second-order MoCo is carried out with the mean height of the antenna footprint for each range and for every pulse instead of using the mean height of the whole scene;

3) same as 2) and applying afterward the proposed subaperture algorithm with a subaperture length of 64 samples and an overlap of $50 \%$.

The observed area has a minimum topographic height of $\sim 550 \mathrm{~m}$ and a maximum of $\sim 1800 \mathrm{~m}$, while the reference height for first-order MoCo is at $1170 \mathrm{~m}$. Platform horizontal and vertical deviations are within \pm 8 and $\pm 4 \mathrm{~m}$, respectively. The technique presented in [1] was used to estimate residual motion errors, which resulted to be less than $\pm 4 \mathrm{~cm}$ in line-of-sight. However, they were not corrected in the presented results so as to validate only the proposed MoCo algorithm. In order to obtain a better calibrated interferogram, [1] should also be applied.

Fig. 3 shows the interferometric coherences for all three cases. Most of the dark areas in Fig. 3(a) and (b) are due to coregistration errors. The left side has worse coherence because the mean height there is quite different from the reference one, and also the topography is steeper, while the right side has less topography variations along azimuth. Fig. 3(c) shows a much better coherence, indicating no azimuth coregistration errors nor phase artifacts exist due to residual phase errors before azimuth compression. Finally, Fig. 4 shows coherence histograms for the three cases plus the backprojected one. Note that the proposed algorithm and the backprojected curves are extremely similar and appear superposed in Fig. 4.

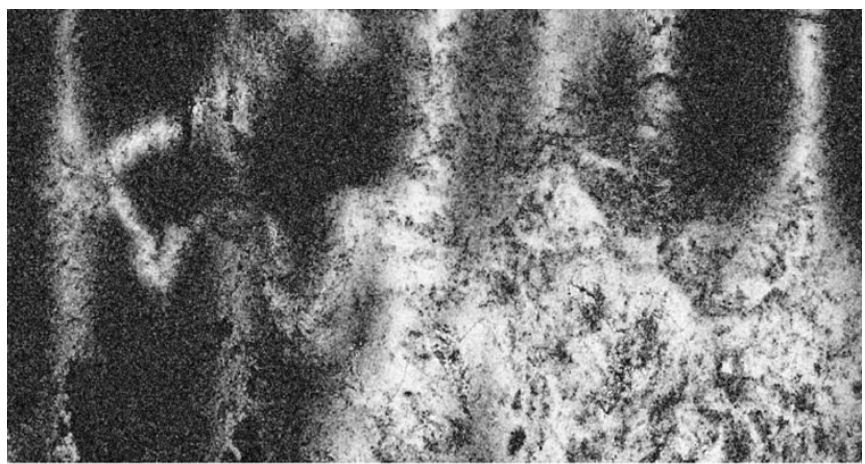

(a)

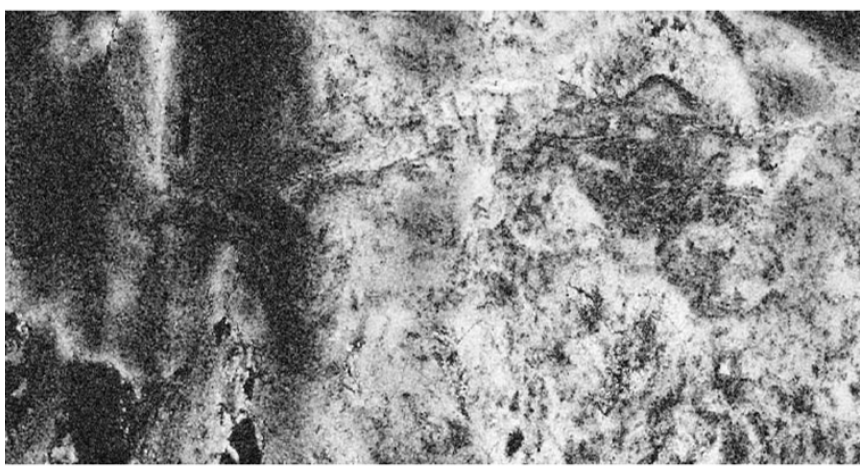

(b)

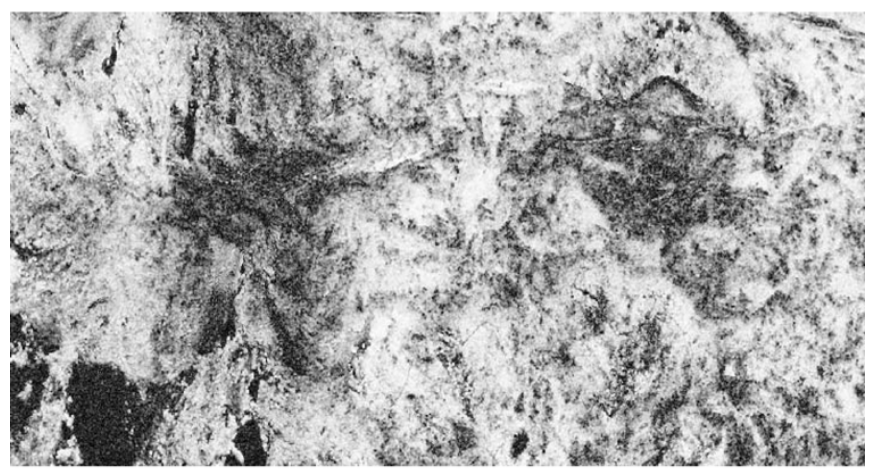

(c)

Fig. 3. (a) Coherence for case 1), (b) for case 2), and (c) for case 3). Coherence values range from black (not coherent) to white (coherent).

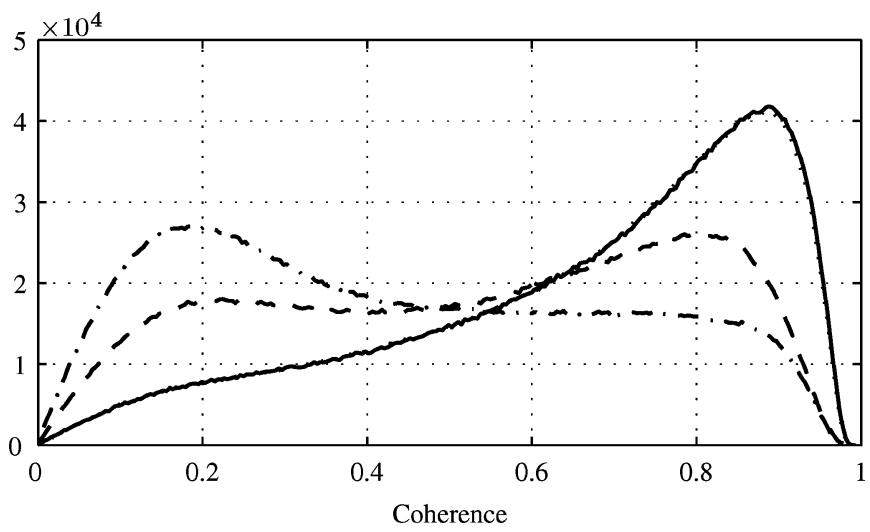

Fig. 4. Coherence histograms. (Dashed-dotted line) Case 1). (Dashed line) Case 2). (Dotted line) Case 3). (Solid line) With backprojection processor.

Additionally, interferograms between slave images for the last two cases and the slave image processed with a conventional 


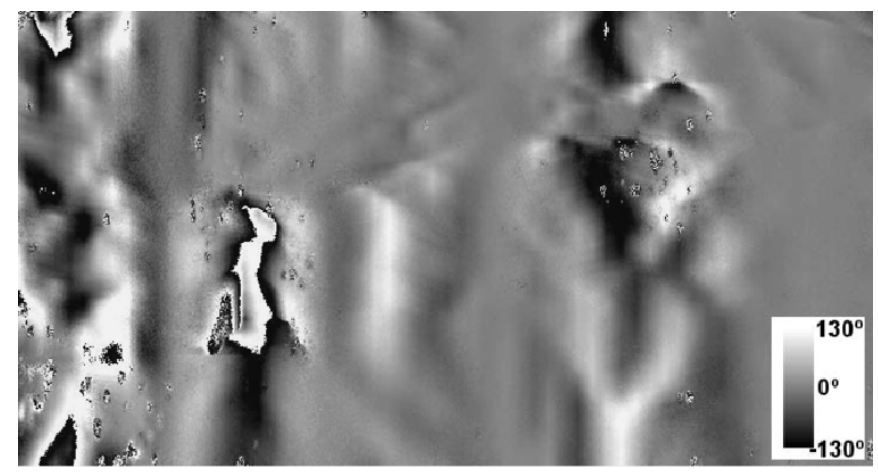

(a)

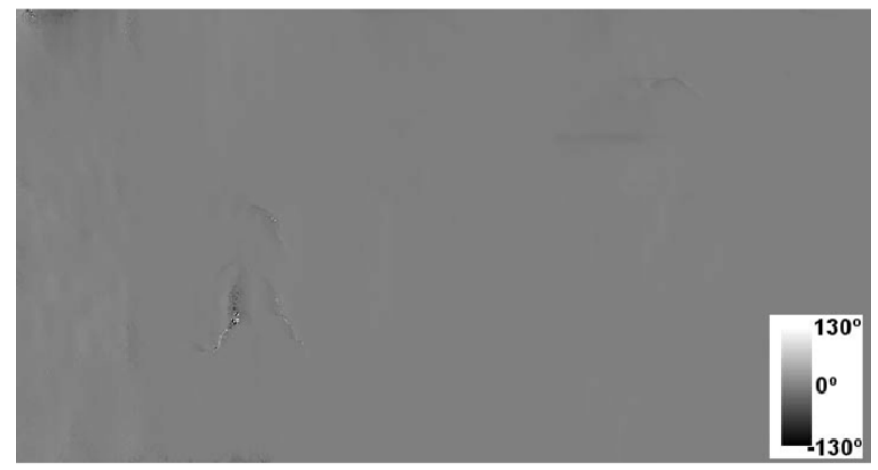

(b)

Fig. 5. Phase error between backprojected slave and slave images for (a) case 2) and for (b) case 3), respectively.

time-domain backprojection algorithm (the ideal solution) have been computed. For case 2), the slave image has been coregistered with the backprojected one. The standard deviation of the phase error with the proposed correction algorithm is less than $3^{\circ}$, while in the other case is $\sim 50^{\circ}$. Fig. 5 shows the phase error for both cases. Note in Fig. 5(a) that in some areas the error is larger than $180^{\circ}$. The performance of the algorithm is independent of the position accuracy of the navigation system, i.e., it will perform almost ideally as shown in Fig. 5(b). Of course, if residual motion errors exist, they will affect in the same way as with conventional MoCo. In that case, [1] should be used to estimate and correct residual motion errors.

Finally, note that the computation time increase is of only $\sim 19 \%$, as the proposed algorithm can be efficiently implemented under a block approach as depicted in Fig. 1.

\section{CONCLUSION}

The proposed algorithm allows to efficiently accommodate strong topography variations during SAR processing when a DEM is available. It has two clear advantages. On the one hand, it allows to obtain an accurate phase, whose accuracy mainly depends on the external DEM used by the algorithm. On the other hand, because it is applied before azimuth compression, it also prevents effects such as defocusing and impulse response displacement, avoiding in the later case the need for azimuthal coregistration. Therefore, it improves the overall quality of the final interferogram in a computationally efficient way. The good performance of the algorithm has been validated with real data.

An important conclusion is that, in airborne repeat-pass systems, when using the proposed MoCo algorithm, there is no need to coregistrate along azimuth dimension, assuming of course that both channels have been processed to have the same final azimuth pixel spacing. Only residual motion errors [1] might remain, which could still induce phase artifacts as well as coregistration errors. However, after applying the proposed MoCo correction, all pixels can be used to precisely estimate residual motion errors according to [1].

Because the proposed algorithm is applied just before azimuth compression, instead of modifying the actual SAR processing scheme, it can also be applied as a postprocessing step. This would require data to be decompressed along the azimuth dimension before correction, and compressed again after applying the proposed correction method.

\section{ACKNOWLEDGMENT}

The authors wish to thank R. Scheiber and K. Macedo for useful discussions, the German Aerospace Center for supplying the E-SAR data, and the NASA/SRTM team for providing the DEM. The authors would also like to thank the anonymous reviewers for their comments and suggestions.

\section{REFERENCES}

[1] P. Prats, A. Reigber, and J. J. Mallorqui, "Interpolation-free coregistration and phase-correction of airborne SAR interferograms," IEEE Geosci. Remote Sens. Lett., vol. 1, no. 3, pp. 188-191, Jul. 2004.

[2] D. R. Stevens, I. G. Cumming, and A. L. Gray, "Options for airborne interferometric SAR motion compensation," IEEE Trans. Geosci. Remote Sens., vol. 33, no. 2, pp. 409-420, Mar. 1995.

[3] R. Scheiber, "A three-step phase correction approach for airborne repeat-pass interferometric SAR data," in Proc. IGARSS, vol. 2, Toulouse, France, Jul. 21-25, 2003, pp. 1190-1992.

[4] A. Potsis, A. Reigber, J. Mittermayer, A. Moreira, and N. Uzunoglou, "Sub-aperture algorithm for motion compensation improvement in wide-beam SAR data processing," IEE Electron. Lett., vol. 37, no. 23, pp. 1405-1407, Nov. 2001.

[5] G. Fornaro, "Trajectory deviations in airborne SAR: Analysis and compensation," IEEE Trans. Aerosp. Electron. Syst., vol. 35, no. 3, pp. 997-1009, Jul. 1999.

[6] A. Reigber, A. Potsis, A. Alivizatos, N. Uzunoglou, and A. Moreira, "Wavenumber domain SAR focusing with integrated motion compensation," in Proc. IGARSS, vol. 3, Toulouse, France, Jul. 21-25, 2003, pp. 2014-2016.

[7] A. Moreira and Y. Huang, "Airborne SAR processing of highly squinted data using a chirp scaling approach with integrated motion compensation," IEEE Trans. Geosci. Remote Sens., vol. 32, no. 5, pp. 1029-1040, Sep. 1994.

[8] R. K. Raney, H. Runge, R. Bamler, I. Cumming, and F. H. Wong, "Precision SAR processing using chirp scaling," IEEE Trans. Geosci. Remote Sensing, vol. 32, no. 4, pp. 786-799, Jul. 1994. 